\title{
Reflexiones en torno a la didáctica latinoamericana: aportes pedagógicos críticos de Paulo Freire y Estela Quintar*
}

\author{
Reflections on Latin American teaching: critical \\ educational contributions of Paulo Freire and Estela \\ Quintar
}

\section{Reflexões sobre o ensino latino-americano: contribuições educacionais críticas de Paulo freire e Estela Quintar}

\author{
Diana Edith Otálvaro Ramírez** \\ Liceo Antioqueño del Municipio de Bello, Antioquia, Colombia \\ Diego Alejandro Muñoz Gaviria*** \\ Universidad de Antioquia, Medellín, Colombia \\ Universidad de San Buenaventura, Medellín, Colombia
}

ReCibido: 2 DE SEPTIEMBRE DE 2013 - APROBAdo: 21 DE OCTUBRE DE 2013

Artículo de investigación, producto del proyecto de investigación, financiado por la Universidad de San Buenaventura, Medellín, titulado: De Tijuana a la Patagonia, perspectivas críticas educativas en América Latina" adelantado por el Grupo Interdisciplinario de estudios pedagógicos (GIDEP). Fecha de inicio julio de 2012; fecha de terminación julio de 2013.

** Licenciada en Música, Magíster en Educación de la Universidad de San Buenaventura y docente de la institución educativa Liceo Antioqueño del Municipio de Bello, Antioquia, Colombia. E-mail: dotalvaro7@hotmail.com

*** Sociólogo, Especialista en Contextualización Psicosocial del Crimen, Magíster en Psicología de la Universidad de San Buenaventura Medellín, con estudios doctorales del Doctorado en Ciencias Sociales: Niñez y Juventud de la Universidad de Manizales y el CINDE, y Estudiante del Doctorado en Filosofía de la Universidad Pontificia Bolivariana. Profesor de la Universidad de San Buenaventura, miembro del Grupo Interdisciplinario de Estudios Pedagógicos de la Universidad de San Buenaventura. También es miembro del Grupo sobre Formación y Antropología Pedagógica FormaF-de la Universidad de Antioquia. E - mail: diegomudante@hotmail.com 
"Actualmente, en la didáctica latinoamericana existe una insuficiente sistematización con respecto a la categorías que debe asumir, lo que ha traído como consecuencia que no siempre se ofrezca a los docentes una posición teórico-metodológica que los oriente en su trabajo diario. En algunos sistemas educativos se importan acríticamente teorías foráneas, sin tener en cuenta la propia realidad educativa. Esto hace, que por ejemplo, en América Latina no esté generalizada aún una verdadera concepción didáctica, elaborada a partir de las sabias experiencias de los educadores latinoamericanos"

(Zilberstein, 2005, p. 32)

Resumen. El presente texto realiza un acercamiento pedagógico, en perspectiva crítica, a las contribuciones que desde América Latina, autores como Paulo Freire y Estela Quintar, brindan al campo de la didáctica. En este sentido, se sustenta la idea de pensar la didáctica crítica de Freire y la didáctica no parametral de Quintar, como didácticas propiamente latinoamericanas.

Palabras clave. Didáctica, didáctica crítica, pedagogía crítica (Tesauro Unesco).

Abstract. This text takes a pedagogical approach in critical perspective to the contributions from Latin America, authors such as Paulo Freire and Estela Quintar, give the field of teaching. In this sense, it supports the Freire's idea of teaching critical and the Quintar's didactics parametral, not as proper American teaching.

Keywords. Didactic, critical didactic, Critical pedagogy (Thesaurus Unesco).

Resumo. Este texto tem uma abordagem pedagógica na perspectiva crítica das contribuições da América Latina, autores como Paulo Freire e Estela Quintar, dar o campo do ensino. Neste sentido, ele defende a idéia de ensinar o pensamento crítico e didática Freire de Quintar Parametral não tão bom ensino Latina.

Palavras chave. Ensino, ensino fundamental, pedagogia crítica (Tesauro Unesco). 


\section{A modo de introducción: el campo disciplinar de las didácticas latinoamericanas}

En el ámbito latinoamericano ha existido históricamente una suerte de campo variopinto o polifónico en torno a la didáctica, lo que otros autores han denominado una diversidad didáctica Brousseau (2007), y esa diversidad básicamente tiene un punto de convergencia, una suerte de atractor del campo, a saber: las preguntas por la enseñanza y las preguntas por lo institucional.

Las ideas en torno a la enseñanza y sus manifestaciones institucionales en américa latina pueden evidenciarse en dos grandes tendencias: en primer lugar, la estela de apropiaciones y recepciones de tradiciones pedagógicas euro centradas, como: la tradición pedagógica anglosajona; la tradición pedagógica francófona; y la tradición pedagógica alemana. Una segunda tendencia en torno a la configuración de una postura didáctica latinoamericana, situada y por ende enraizada en las problemáticas pedagógicas, políticas y culturales de esta región.

En el caso de la recepción o apropiación de las didácticas euro centradas, estudios en américa latina como el de Caruso y Dussel (2006), el de Zuluaga (1999) y el de Sáenz y otros (1997), permiten comprender y reconstruir las maneras en que las posturas didácticas extranjeras han llegado a nuestro continente con unos intereses de colonialismo académico, político y cultural que son funcionales a las propuestas civilizatorias coloniales. En este caso, lo didáctico, tal como lo veremos al final del texto, asume una postura parametral, es decir, asume un interés técnico que reduce el tema de la enseñanza a reproducción civilizatoria de las formas de colonialidad ofrecidas desde las metrópolis y sus pensadores. En este sentido, los trabajos de Ribeiro (1993) y Castro (2000) son seminales en la exploración de la conexión política y pedagógica de las formas de enseñanza importadas a la región y las maneras de colonialidad del ser, el saber y el poder que han sufrido los pueblos latinoamericanos.

Pensar el campo disciplinar de la didáctica en latino américa desde esta primera tendencia, equivale a ver la didáctica en la región como un "préstamo" educativo de las diferentes tradiciones pedagógicas que han tocado desde sus perspectivas culturales, esquemas de pensamiento, intereses políticos y demás, el tema didáctica con diferentes intencionalidades.

ITINERARIO EDUCATIVO • ISSN OICI-2753 • AÑO XXVII, N. ${ }^{\circ} 62$ • JULIO - DICIEMBRE DE 2OI3 • P. 43-58 
Ahora, para el caso del campo disciplinar de la didáctica latinoamericana el problema fundamental no es la mentalidad que trae la tradición, el problema se encuentra en por qué esa mentalidad que trae la tradición es apropiada o recepcionada en nuestro contexto, en otras palabras, por qué es importante la circulación o por qué se dio la circulación de ciertas concepciones de la didáctica en los contextos latinoamericanos.

En esa perspectiva, hay presencia de la mirada anglosajona de la didáctica, haciendo la salvedad o advertencia que hablar de didáctica en dicha mirada es arriesgado por su "estigmatización", por su falta de cientificidad y por un fuerte entramado normativo o moralizante.

No obstante, en el ámbito latinoamericano desde la década de los años setenta del siglo pasado, viene tomando fuerza una diálogo entre lo que para América Latina se ha denominado didácticas de las ciencias, principalmente la didáctica de las matemáticas, y los estudios de corte anglosajón que se han preguntado por los estudios científicos sobre la enseñanza de las matemáticas o sobre la enseñanza de las ciencias.

En ese sentido, hay un diálogo y tensión entre estudios de la enseñanza y aprendizaje, y algunas tensiones conceptuales después de la década de los años ochenta del siglo pasado, entre perspectivas conductistas clásicas y perspectivas, que se han autodenominado, del constructivismo radical.

Un texto importante para reconstruir este proceso de apropiación anglosajona es el del profesor mexicano Ángel Díaz Barriga (1984), La formación de profesores, un problema estructural; en éste se logra ubicar un poco la tensión entre la manera como se entienden las didácticas de las ciencias en América Latina y la perspectiva de los estudios científicos en educación sobre la enseñanza de ciencias anglosajonas.

Desde otro punto de vista se encuentra la mirada francófona, con estudios que se han realizado en América Latina, específicamente en Colombia, que focalizan su interés en las perspectivas didácticas que han circulado a nivel institucional en las instituciones formadoras de maestras y maestros. La tesis fundamental es que su presencia tiene relativa visibilidad en la historia latinoamericana entre los años treinta y los sesenta del siglo pasado; con un supuesto que reduce la concepción de la didáctica a un conjunto de métodos, técnicas y procedimientos para la enseñanza. 
En esta orientación la didáctica queda reducida a un saber instrumental de la enseñanza. Es importante reconocer el texto de la profesora De Tezanos (2006), Didáctica-pedagogía-ciencia de la educación: la relación que confirma la "excepción" francesa, un texto que permite evidenciar la concepción francófona de la didáctica y sus implementaciones.

Por último, la presencia de la perspectiva alemana, que ha tenido diferentes misiones pedagógicas al contexto latinoamericano. En este aspecto es posible citar un trabajo que se llevó a cabo en conjunto entre pedagogos chilenos y pedagogos alemanes, intentando identificar modelos didácticos provenientes de Alemania que han tenido presencia en América Latina, ante lo cual se identificaron veinte modelos traídos de teorías alemanas que habían circulado en América Latina, quizá uno de los más famosos es el seminario alemán.

En resumen, con esta primera tendencia en la configuración del campo didáctico latinoamericano, el centro del estudio está en el tipo de saberes y prácticas que en torno a la didáctica circularon y circulan en américa latina, con la consecuente pregunta por las divergencias y convergencias entre los contextos de producción del saber didáctico, y los contextos de apropiación o recepción. Así, lo interesante de esta perspectiva radica en la identificación, reconstrucción y propuesta de rejillas de apropiación que sean capaces de colocar el acento en las maneras en que desde los contextos de recepción de los saberes didácticos, éstos sufren transformaciones, modificaciones o cambios. Sin embargo, la crítica hecha a este enfoque, es que sólo se queda en la lectura de la reproducción o de la resistencia, dejando por fuera, lecturas que reivindiquen la producción o construcción propia.

En el segundo sentido, es decir desde la producción "situada" de la didáctica en américa latina, con autores como Freire (1970, 1971), Díaz Barriga (1984), Zemelman (1998) y Quintar (2008), entre otros, es posible decir que hay una emergencia y un atisbo de la didáctica latinoamericana después de la década de los años cincuenta del siglo pasado, en la perspectiva inicial de una didáctica en la obra de Paulo Freire y sus planteamientos en torno al debate de la educación bancaria y la reivindicación de una enseñanza crítica. No obstante, es importante hacer una salvedad, en las obras de Freire no se ha encontrado el enunciado explícito de didáctica, se encuentra el enunciado de una enseñanza crítica o la educación como prácticas de liberación, pero el sentido didáctico 
a partir de la pregunta por la enseñanza y sus necesarios vínculos con lo político están en la base del pensamiento freiriano. Para este autor, la enseñanza que está en la base de una educación liberadora, es una enseñanza crítica.

Aunque hay trabajos posteriores que reconstruyen bajo el concepto de didáctica algunos supuestos freirianos que podrían dar luces para pensar una didáctica latinoamericana. En este sentido se pueden citar dos textos: el primero del profesor Torres Carrillo (2001), Ires y venires en la educación popular en América Latina, donde ubica la obra de Freire como un iniciador de la didáctica crítica latinoamericana. Un dato curioso en este aspecto es que Freire es uno de los autores citados por los teóricos críticos del currículo anglosajón para la configuración de lo que posteriormente en Estados Unidos se va a enunciar como teoría crítica y pos-crítica del currículo (Giroux, 2003 y Mclaren, 1994).

El segundo texto del profesor Marco Raúl Mejía (2011), titulado Educaciones y pedagogías críticas desde el sur. En este escrito el profesor logra ubicar la obra de Freire como base del pensamiento pedagógico crítico latinoamericano.

Ahora bien, algo que viene circulando con gran fuerza desde el 2000 en el ámbito latinoamericano en México, Argentina, Brasil y en Colombia es lo que se ha denominado como didácticas no parametrales, en este tema es importante la obra de Hugo Zemelman y la influencia de este autor sobre el pensamiento filosófico latinoamericano, la pregunta por el sujeto latinoamericano, lo que él denomina como una pedagogía de la potencia y una de las pedagogas que ha estado cercano al pensamiento de Zemelman, la profesora Estela Quintar (2008) en su texto Didáctica no parametral: senderos hacia la descolonización.

En esta segunda tendencia de la configuración del campo didáctico latinoamericano, lo central en el estudio es la producción propia, la forma en que desde nuestros contextos, situaciones y realidades configuramos praxis didácticas capaces de responder las problemáticas pedagógicas y políticas de la región. Es importante resaltar, que las ideas de los pedagogos y pedagogas sobre el tema, encuentran un lugar común en el pensamiento crítico. Pensar pedagógica y didácticamente américa latina 
exige una orientación crítica, una visión que permita despertar de las formas de colonialidad existentes.

Para la orientación asumida en este texto, es fundamental la configuración del campo didáctico latinoamericano desde la perspectiva de los estudios, comunidades y pensadores que focalizan su atención en las maneras propias - situadas de asumir pedagógica y políticamente la enseñanza y sus correlatos institucionales.

Por lo anterior, se procederá a continuación a exponer algunas de las ideas pedagógicas y políticas que han permitido en las voces de Paulo Freire y Estela Quintar, reivindicar una didáctica latinoamericana de vocación crítica.

\section{Contribuciones freirianas a la didáctica latinoamericana}

Recuperar a Freire para una didáctica crítica supone insertar el debate didáctico en el horizonte del pensamiento latinoamericano, es decir, mirar, problematizar y sentirnos ocupados en el escenario de la adversidad de nuestro continente. Esta didáctica se legitima pedagógicamente en el contexto de la pedagogía del oprimido de Freire, o en un sentido más amplio, de la educación popular latinoamericana.

Lo anterior se convierte en un filtro pedagógico y político, frente a la oleada de perspectivas didácticas instrumentales, las posturas latinoamericanas se sitúan en una lectura explícitamente crítica. Desde la herencia freiriana, no podría pensarse la enseñanza desprovista de la pregunta antropológica y pedagógica por el tipo de ser humano y de sociedad allí presente, y por sus conexiones o desconexiones con las realidades situadas o contextuales. Por lo anterior, en la lectura latinoamericana de la didáctica, la enseñanza no se reduce a mera instrucción, el ser humano a especulación metafísica, y la sociedad a parámetros. Por el contrario, la enseñanza es intersubjetividad, el ser humano una biografía, y la sociedad una construcción colectiva.

Desde la pedagogía de Paulo Freire, la didáctica latinoamericana se reivindica como un saber crítico y situado, una praxis pedagógica y política, que sin desconocer los aportes y las posturas configuradas en otras latitudes, se ocupa de las problemáticas específicamente latinoame-

ITINERARIO EDUCATIVO • ISSN OIZI-2753 • AÑO XXVII, N. ${ }^{\circ} 62$ • JULIO - DICIEMBRE DE ZOI3 • P. 43-58 
ricanas, es decir, de las situaciones de opresión generadas en la realidad política de la región.

Una vez expuesto lo anterior y con relación a los aportes de la obra de Freire a la didáctica, es pertinente preguntarse: ¿Cuáles son las contribuciones pedagógicas freirianas a la didáctica latinoamericana?

Diferentes autores latinoamericanos han estado en la pesquisa de intentar reconstruir supuestos de la obra de Freire que aún tienen presencia en la actualidad, ya que aún son pertinentes para pensarnos como latinoamericanos (as). En este sentido es pertinente citar los trabajos del profesor Torres (2008), en los cuales se deja clara la base freiriana de la educación popular latinoamericana. Los estudios de Rubens (2009), quien siendo discípulo de Freire logra evidenciar la pertinencia y actualidad de su pedagogía para pensar temas políticos de la resistencia y la transformación social. El trabajo de Escobar (2005), quien logra desde una lectura freiriana al amor y el cambio, ver las conexiones de la pedagogía del oprimido con movimientos sociales como el movimiento zapatista en México. Estos y otros estudios, permiten defender la actualidad y presencia del pensamiento pedagógico y político del autor en la compleja realidad latinoamericana. Para esta comunidad de educadores (as) populares o pedagogos (as) críticos, las contribuciones pedagógicas freirianas a la didáctica latinoamericana, podrían reconstruirse en torno a los siguientes aspectos: en primer lugar y como aspecto político crítico, se encuentra la defensa de la formación humana como emancipación; en segundo lugar, la recuperación de la voz de cada sujeto, su auto narrativa como defensa del ser - sujeto; en tercer lugar, la comprensión de la alfabetización como una acción pedagógica - política tendiente a la concienciación, no a la adscripción instrumental a un universo vocabular; el cuarto aspecto es el reconocimiento de la práctica de enseñanza como una práctica política interesada; el quinto tema sería la centralidad del diálogo como vinculante social; por último, la pedagogización del mundo como lectura pedagógica crítica a la realidad histórica vivida. A continuación se tematizarán cada una de estas contribuciones.

La primera contribución desde la pedagogía freiriana a la didáctica, quizás la fundamental, es la reivindicación de la centralidad pedagógica y política de la formación como emancipación. Para Freire (pedagogía de la autonomía), el ser humano es un ser en proceso, que en su devenir va configurando prácticas, experiencias y reflexiones que le permiten 
emanciparse. Aquí, la emancipación trasciende su reducción política de adscripción, inserción, reconocimiento, suministro, para llegar a ser, en clave de Marx, una real emancipación humana, esto es, un reconocimiento y defensa de la capacidad humana de hacerse, haciendo el mundo en relación con los otros (Marx, 2009). Lo central de comprender la formación como emancipación, radica en la perspectiva pedagógica y política crítica que reivindica como centralidad de todo acto educativo y de la enseñanza, la contribución antropológica al llamado histórico del ser humano de ser más (Freire, 1971, p. 31). Ser más en Freire, es emanciparse humanamente, es hacer de sí una tarea permanente, a lo cual debe responder la enseñanza. Lo didáctico en este sentido tiene una base formativa insoslayable, no podría pensarse la didáctica sin una fundamentación formativa que en clave crítica, tienda o permita la emancipación humana.

La segunda contribución se centra en la recuperación de la narración de los sujetos. Estas narraciones evidencian la comprensión de sus experiencias en un devenir histórico anclado en el marco de las luchas sociales. Narrarse es hacer de sí una tarea, es tomar conciencia del trayecto existencia que cada uno y cada una representa. La auto narración permite didácticamente, la defensa política de la condición humana del sujeto, que en tanto tal, rompe con su reducción a objeto, recipiente o tabula rasa, para asumirse como creador, autor y agente de sus propias existencias. Esta contribución a la didáctica descentra la pregunta por la enseñanza de la instrumentación, para darle la trascendencia de recuperador de la existencia, de allí su conexión con el tema de la emancipación humana (Freire, 1970).

Las anteriores contribuciones están en la base de la propuesta pedagógica de Freire en torno a la alfabetización. Para el autor alfabetizar es concienciar, es tomar conciencia de la condición de ser humano - sujeto que rompe con las reducciones bancarias - técnicas del ser humano objeto. Por lo anterior, la propuesta de alfabetización de Freire no es una adaptación funcional a un determinado universo vocabular, es más bien, la inserción crítica a dicho universo, con lo cual el sujeto ve la importancia de sus praxis vitales, de sus agencias, de sus posturas, de sus voces (Freire, 1971, p. 99). Así, para Freire, en clave de una didáctica crítica latinoamericana, la lectura y la escritura no son importantes si primero no atraviesan la pregunta por el sujeto y por el mundo en el cual ese sujeto habita, es decir, escribir sólo es pertinente en cuanto escribirse y leer en tanto leerse (Freire,1971, p.110). 
Un elemento más, es comprender que la enseñanza es una práctica educativa y que ésta es una práctica social ideologizada, es decir, no se podría llegar a la enseñanza con la ingenuidad de creer que es neutral, desprovista de intereses, que no pertenece a un contexto, que no hay ideología o que no está inmerso en una suerte de poderes políticos. Por ello, para la didáctica latinoamericana será pertinente enunciar abiertamente su fundamentación crítica, sus propuestas de enseñanza son reconstructivas y problematizadoras de la realidad, la sociedad y la existencia, por ello la enseñanza se desmarca de la instrucción para fundamentarse intersubjetivamente, en las relaciones humanas.

De otro lado, los temas generadores de esta perspectiva Freiriana tienen que ver con el diálogo, la pregunta, el partir de la experiencia cultural, la politización de la enseñanza, el carácter liberador de la enseñanza (Freire, 1971). Estos temas generadores exceden una propuesta metodológica - técnica, y en la perspectiva latinoamericana se concentra fundamentalmente en una apuesta política por otros mundos posibles (Freire, 1993).

Por último, y en esta lectura lo más importante, en la contribución pedagógica Freiriana para una didáctica latinoamericana es la expresión de la pedagogización del mundo, y no se trata de la pedagogización en el sentido de creer que todo es posible de ser enseñado o aprendido, esa pedagogización bajo la perspectiva de Paulo Freire es una praxis pedagógica y política que reivindica en todo tiempo y espacio, la configuración de sujetos autónomos que buscan la emancipación, la idea antropológica de seres humanos en búsqueda de la libertad (Freire, 1970).

Una vez expuestas, grosso modo, estas contribuciones pedagógicas freirianas a la didáctica latinoamericana, se hace pertinente recuperar la voz de una de las pedagogas que en la actualidad ha propuesto una manera concreta de enunciar esa didáctica crítica latinoamericana de base freiriana, ella es la profesora Estela Quintar y sus ideas en torno a la didáctica no parametral.

\section{La propuesta didáctica de la profesora Quintar: la didáctica no parametral}

Es importante recordar que la obra de la profesora Quintar se encuentra inmersa en el campo académico y político del Instituto de Pensamiento y Cultura Latinoamericana (IPECAL), coordinado por el profesor Hugo 
Zemelman y apoyado académicamente por pensadores latinoamericanos como Enrique Dussel, Anibal Quijano y Boaventura de Sousa. El IPECAL tiene en sus sustentos pedagógicos y políticos un fuerte diálogo con la propuesta Freiriana, en temas como: pensar lo latinoamericano en la línea del pensamiento crítico; defender la pertinencia de configurar propuestas pedagógicas y políticas situadas, enraizadas en las realidades socio históricas; la reivindicación de la capacidad de agencia de los sujetos educativos; la perspectiva esperanzada o de optimismo crítico en torno a la educación y la formación; entre otros temas.

La profesora Quintar desde sus diálogos con Freire y Zemelman, logra proponer una manera crítica latinoamericana de pensar y actuar en torno a la didáctica. Para la profesora lo didáctico, como todo lo humano, tiene una base existencial, no sería posible hablar de la didáctica sin el reconocimiento del ser humano. Y por lo anterior, el tema de la enseñanza no sería posible sin la experiencia humana. En palabras de la profesora Quintar (2008):

lo educativo emergió como una posibilidad de lucha y resistencia por la voz, por el derecho a pensar, por la necesidad existencial de ser autónomos y poder elegir como ser y vivir ese ser, por la identidad y la memoria que nos permite mayor conciencia histórica y sentido de futuro. En este sentido la enseñanza como espacio esperanzado y como promesa constante desde donde poder contribuir al cambio de nosotros mismos para cambiar nuestro entorno, y desde allí nuestras sociedades, pasó a ser un proyecto de sentido y de vida. Y sigo convencida de esta opción, convencida de que si no cambiamos las personas no cambiará el sistema, por más reformas curriculares o educativas que escribamos en los papeles, somos las personas las que hacemos a los cambios y no los decretos (p.28).

Las didácticas que no reconocen su base antropológica, son didácticas parametrales, un tipo de saber pedagógico al servicio de un saber político de la reproducción, que sólo se interesa por la sofisticación instrumental - técnica de las estrategias tendientes a la masificación y alienación cultural. Para las didácticas parametrales, la particularidad del ser humano no interesa, de forma bancaria, centran sus esfuerzos en la adaptación social y en la reducción del ser humano a ser - objeto. Lo parametral es lo establecido, lo creado, lo estandarizado, cierto tipo de recetario, quien se preocupe por este tema verá en lo pedagógico y didáctico una excusa para la repetición y conservación. 
En contradicción con lo parametral, lo no parametral centra su interés en la resistencia, la transformación, la alteración y la producción. Romper con los parámetros, trascender los límites, ir más allá de los linderos, será la vocación histórica de esta postura pedagógica y política. La didáctica no parametral es totalmente coherente con el llamado antropológico y político de Freire, en cuanto reconocimiento del ser humano como un ser llamado a ser más. Y con la propuesta filosófica de Zemelman (1998) del sujeto potente, que se desmarca de los parámetros que intentan condicionar su existencia.

Por lo anterior, la didáctica no parametral es una didáctica crítica pensada desde Latinoamérica, que permite desde Tijuana hasta la Patagonia praxis educativas críticas, de allí que sus preguntas centrales sean:

¿Qué es lo que somos como seres humanos en América Latina y en cada uno de nuestros países? ¿Quién nos dice como tenemos que actuar subjetiva y socialmente? ¿̇En qué momento la perdida de la voz comenzó a garantizar la vida? ¿Cuáles son las inercias socioculturales que legitiman creencias que justifican el "no ver-nos" en nuestras propias prácticas y relaciones? ¿Qué aspectos psico-cognitivos bloquean en América Latina la capacidad de preguntar con sentido de futuro? ¿Por qué nos resignamos ante lo que no es posible resignarse? ¿Por qué nos acomodamos tan fácilmente a lo que nos duele como sujetos y como países? ¿Cómo hacer de la vida cotidiana un espacio de permanente darse y dar cuenta de donde, por qué y para qué se vive subjetiva y colectivamente? ¿Qué es lo que podemos hacer para ir legitimando día a día la muerte, la locura y la violencia de la exclusión por raza, clase o etnia o bien la violencia sutil y cotidiana de la pérdida del propio sentido de vida? (Quintar, 2008, p. 30).

Las anteriores son preguntas que visibilizan el sentido de una didáctica crítica a la latinoamericana. Recordando las ideas expuestas en la introducción, el campo disciplinar de la didáctica ha tenido dos preguntas centrales: la pregunta por la enseñanza y la pregunta por sus correlatos institucionales. A continuación se harán algunas reflexiones acerca de la manera en que la didáctica no parametral enfrenta estos temas.

El ¿Para qué enseñar? desde la perspectiva didáctica no parametral, se responde las siguientes maneras: 
Primero, porque la educación es una opción ético-política, en esto hay una congruencia con la lectura Freiriana. Enseñar no es reproducir, es suscitar la intersubjetividad, el diálogo y con ellos la transformación, el cambio.

Segundo porque se pretende promover sujetos autónomos - potentes, que en sus procesos de alfabetización - concienciación asuman compromisos con sus existencia. Este tipo de sujetos potentes pueden desalojar al opresor que llevan dentro (Freire, 1970) o reivindicarse como constructores de realidad (Zemelman, 1998).

Y por último, aportar a una pedagogía de la potencia y no, como lo expresa la profesora Quintar, a una pedagogía del bonsái, la pedagogía de las ideas cortas, pequeñas, minúsculas que se guían por parámetros coloniales. Esta pedagogía pretende invisibilizar la potencia humana, su energía constructora a través del uso repetitivo de formas de "poda", de corte de las ideas y las prácticas que permitan expandir la existencia humana y con ella, el tipo de sociedades que construimos.

Otro cuestionamiento pertinente en la perspectiva no parametral de la didáctica es: ¿Qué tipo de configuración institucional asume la enseñanza? en este aspecto nos ofrece la profesora Quintar las siguientes ideas:

En primera instancia, una crítica radical, siendo esta la herencia Freiriana a la educación popular que también la retoma la didáctica no parametral. Aquí lo radical insinúa una comprensión del ser humano como ser comprometido con su propia existencia, y por ello, sensible y solidario con la existencia de los otros y de lo otro (Freire, 1971, p. 41) Para Freire lo radical se diferencia de lo sectario en cuanto el sectarismo es la adopción dogmática de una idea, que permite como evidencia existencial negar la propia subjetividad en aras de defender e imponer una determinada ideología. Lo radical es una responsabilidad del sujeto con su propio mundo de la vida.

En segundo momento, una crítica ideológica a la escuela como una institución del sin sentido, como una institución que agencia procesos de colonización. Con esta crítica, se logra reconstruir la institución escuela y desprender de ella los mitos modernos que le han hecho ver natural, por ejemplo: el mito del progreso a ella indexado, el mito de la compe- 
tencia como base de la relación social, el mito de la clasificación, entre otros. Una vez desnaturalizada la escuela, tanto ésta como otras formas de concretar la educación, pueden abrir el horizonte de la creatividad y la transformación institucional.

Otro aspecto relevante es la concepción del espacio pedagógico no sólo como un espacio escolar donde lo educativo emerge como posibilidad de lucha, trascendiendo sus reducciones, a mera reproducción. Para la didáctica no parametral, el espacio pedagógico es un espacio potenciador del sujeto, que en determinadas situaciones contradice la lógica y la propuesta de la escuela como espacio de instrucción o adoctrinamiento.

En última instancia, se trata de entender que la didáctica no parametral propone la formación de sujetos críticos y creativos capaces de pensar y actuar en su contexto histórico. No se ve en el sujeto una adaptación al entorno, se ve en el sujeto una inserción crítica que le permite trascender los hechizos del entorno.

\section{A modo de cierre}

La didáctica latinoamericana expresa una gran complejidad, evidenciada en sus configuraciones tendientes a la reproducción de enfoques y tradiciones pedagógicas foráneas, y sus configuraciones basadas en la producción propia y situada. En este texto se ha querido reivindicar como postura didáctica propiamente latinoamericana, la didáctica crítica que enfrenta las situaciones de opresión de nuestra región.

La didáctica latinoamericana es una didáctica crítica, que coloca como centro de la enseñanza y la institucionalidad, la defensa de la emancipación humana, rompiendo así, con los reduccionismos bancarios que han querido ver la enseñanza y sus correlatos institucionales, como estrategias de reproducción de los parámetros culturales, sociales y políticos sustentados por ciertos grupos o clases sociales.

En esta perspectiva las voces de autores y autoras como Paulo Freire y Estela Quintar, permiten la construcción de un campo disciplinar de la didáctica crítica latinoamericana. Para estos autores la radicalidad de la didáctica crítica latinoamericana se expresa en la idea antropológica, pedagógica y política del ser humano como sujeto potente, como ser 
llamado históricamente a ser más. Por lo anterior, esta didáctica es una perspectiva esperanzadora que de forma optimista, es decir creativa o propositiva, critica las realidades y relaciones de opresión existentes en la región.

Queda aún por tematizar, las maneras en que esta didáctica crítica latinoamericana, freiriana y no parametral, se concretan en medios, mediaciones, interacciones, escenarios y subjetividades concretas. Es pertinente en este sentido, posteriores ejercicios de reflexión que posibiliten, la reconstrucción y sistematización de experiencias didácticas latinoamericanas que permitan tornar realidad existente, las ideas de Freire y Quintar.

\section{Referencias}

Brousseau, G. (2007). Iniciación al estudio de las teorías de las situaciones didáctica. Buenos Aires: Libros del Zorzal.

Caruso, M. y Dussel, I. (2006). La invención del aula. Una genealogía de las formas d enseñar- Buenos Aires: Santillana.

Castro, S. (2000). Ciencias sociales, violencia epistémica y el problema de la "invención del otro". En La colonilidad del saber: eurocentrismo y ciencias sociales. Buenos Aires: Clacso.

De Tezanos, A. (2006). Didáctica-pedagogía-ciencia de la educación: la relación que confirma la "excepción" francesa. En Revista Educación y Pedagogía, Medellín, Universidad de Antioquia, Facultad de Educación, vol. XVIII, núm. 46, (septiembre-diciembre), pp. 33-57.

Díaz Barriga, A. (1988). La formación de profesores, un problema estructural. En Revista Foro Universitario, núm 48. México: STUNAM.

Escobar, M. (2005). Pedagogía erótica, Paulo Freire y el EZLN. México: UNAM

Freire, P. (1970). Pedagogía del oprimido. Buenos aires: Siglo XXI editores.

Freire, P. (1971). La Educación como práctica de la libertad. Buenos aires: Siglo XXI editores.

Freire, P. (1993). Pedagogía de la esperanza, un reencuentro con la pedagogía del oprimido. Buenos Aires: Siglo XXI editores.

ITINERARIO EDUCATIVO • ISSN OIDI-2753 • AÑO XXVII, N. ${ }^{\circ} 62$ • JULIO - DICIEMBRE DE 2OI3 • P. 43-58 
Giroux, H. (2003). Pedagogía y política de la esperanza. Teoría, cultura y enseñanza. Buenos Aires - Madrid: Amorrortu editores.

Marx, K. (2009). La cuestión judía. México - Barcelona: Anthropos.

Mclaren, P. (1994). Pedagogía crítica, resistencia cultural y la producción del deseo. Buenos Aires: Cuadernos.

Mejía, M. (2011). Educaciones y pedagogías críticas desde el sur. Bogotá: Magisterio Editorial.

Quintar, E. (2008) Didáctica no parametral: senderos hacia la descolonización. México: IPECAL.

Ribeiro, D. (1993). El proceso civilizatorio. Cali: Universidad del Valle.

Rubens, J. (2009). Apuntes bio - bibliográficos. Bogotá: Magisterio editorial.

Saénz, J., Saldarriaga, O. y Ospina, A. (1997). Mirar la infancia: pedagogía, moral y modernidad en Colombia, 1903 - 1946. Tomo II. Medellín: editorial Universidad de Antioquia.

Torres, A. (2001). Ires y venires en la educación popular en América Latina. En: Revista La Piragua, número 50- marzo 2001.

Torres, A. (2008). Educación popular, trayectoria y actualidad. Caracas: Imprenta UBV.

Zemelman, H. (1998). El conocimiento como desafío posible. México: colección conversaciones didácticas.

Zilberstein, T. (2005). Aprendizaje, enseñanza y educación desarrolladora. México: CEIDE.

Zuluaga, O. (1999). Pedagogía e historia. La historicidad de la pedagogía, la enseñanza, un objeto de saber. Barcelona: Anthropos. 